Vol. 1, No. 1, 2016

\title{
ERRORS OF PHASE SHIFT MEASURING BY ALGORITHMIC SUM-DIFFERENCE METHODS FROM AMPLITUDES INEQUALITY AND THEIR REDUCTION METHODS
}

\author{
Ihor Buchma \\ Lviv Polytechnic National University, 12, Bandera Str., Lviv, 79013, Ukraine \\ Author e-mail: ibuchmal@gmail.com
}

Submitted on 29.09.2015

(C) Buchma I., 2016

Abstract: This paper analyzes the errors entailed by the signal amplitude inequality when measuring the phase shift among low frequency harmonic signals for algorithmic sum-difference methods. It is shown that additional phase shifts of summary and difference signals caused by the inequality of compared signals in some methods affect the errors, whereas in others they do not. A method in which there is no additive component of the error has been found. This method has been admitted to be the best to measure small phase shifts.

The method for measuring the phase shift, which enables to avoid errors due to amplitude inequality of compared signals has been proposed.

Key words: errors, harmonic signals, signal amplitude inequality, phase measuring, low frequency, sum-difference method, amplitude errors.

\section{SYMBOLS}

$U_{m}, \varphi_{x}, \omega$ - amplitude of signals with equal amplitudes, phase shift and its angular frequency accordingly; $U_{R m}, U_{\Sigma m}$ - amplitude of difference and summary signals accordingly; $\delta U=\frac{\Delta U}{U_{m}}=\frac{U_{2}-U_{1}}{U_{1}}=\frac{U_{2}-U_{m}}{U_{m}}=$ $=\frac{\Delta U}{U_{m}}-$ relative amplitudes difference of signals with unequal amplitudes $U_{1}$ and $U_{2} ; \Delta U=U_{2}-U_{1}-$ absolute amplitude difference of signals; $t_{1}$ - the moment when the sum of signals compared by phase with equal amplitudes goes through zero; $t_{2}$ - the moment when the difference of signals compared by phase with equal amplitudes goes through zero; $t_{1}^{\prime}-$ the moment when the sum of signals compared by phase with unequal amplitudes goes through zero; $t_{2}$ - the moment when the difference of signals compared by phase with unequal amplitudes goes through zero; $t_{0}-$ the moment when the instantaneous value of the second signal $u_{2}\left(t_{0}\right)$ is zero; $\Delta \varphi$ - absolute error of phase measurement; $\delta \varphi-$ relative error of phase measurement; $K$ - coefficient of boosting amplifier; $\Delta K$ - absolute error of boosting amplifier coefficient; $k$ - constant equal to boosting amplifier coefficient $K$.

\section{INTRODUCTION}

The eddy-current measurement and computational tools of cyber-physical systems [1] are based on measuring small phase shifts. In particular, phase methods are widely used in control systems, such as radar [2], systems of diagnostic of steel sheet structures corrosion by eddy current method $[3,4]$ and others. In radar systems and systems of inspection of steel sheet structures corrosion measurement of small phase shifts between two low-frequency harmonic signals is required [2-4]. Thus, it may be concluded that phase shifts measurements are widely used in science and technology.

In automatic control systems during measuring the phase shift between low-frequency signals the speed of measurement tools is important. The Increase of phase shift measurement speed was achieved by going from analog to digital methods.

Therefore, algorithmic methods for phase shift measuring became widespread with the development of Computers and analog-to-digital converters. These methods provide high speed of operation as they allow to obtain a measurement result during the time which is not bigger than half-period signals [5,6]. This is especially important in the range of infra-low frequencies, where the speed of operation is a crucial parameter.

Methods with sum-difference transformation are distinguished among algorithmic methods of phase shift measuring owing to their simplicity and high sensitivity. However, these methods provide sufficient accuracy 
when amplitudes of signals are equal. Equalization of signal amplitude is not an easy task. It demands additional algorithmic and hardware costs.

\section{PUBLISHED DATA REVIEW}

Let us examine these methods. To do this we will take two harmonic signals with equal amplitudes and different initial phases. Let us assume that the first signal initial phase equals zero, and the second outgoes the first one by angle $\varphi_{x}$.

Sum-difference methods are based on the creation of difference and summary signals.

As shown in [4], in this case the difference signal $u_{R}(t)$ is described by the following expression

$$
u_{R}(t)=2 U_{m} \sin \frac{\varphi_{x}}{2} \cos \left(\omega t+\frac{\varphi_{x}}{2}\right),
$$

and the summary one by $\mathrm{u}_{\Sigma}(t)$ - accordingly

$$
u_{\Sigma}(t)=2 U_{m} \cos \frac{\varphi_{x}}{2} \sin \left(\omega t+\frac{\varphi_{x}}{2}\right) .
$$

At the moment $t_{l}$ of summary signal $u_{\Sigma}(t)$ transition through zero the signal $u_{R}(t)$ equals amplitude value, that is

$$
u_{R}\left(t_{1}\right)=U_{R m}=2 U_{m} \sin \frac{\varphi_{x}}{2} .
$$

At the moment $t_{2}$ when the difference signal goes through zero the summary signal equals the amplitude value

$$
u_{\Sigma}\left(t_{2}\right)=U_{\Sigma m}=2 U_{m} \cos \frac{\varphi_{x}}{2} .
$$

In [4] it is shown that by measuring amplitude of difference signal at the moment $t_{1}$, and the summary signal - at the moment $t_{2}$, we receive several dependences to determine the phase shift $\varphi_{x}$-namely:

$$
\begin{gathered}
\varphi_{x}=2 \operatorname{arctg} \frac{U_{R m}}{U_{\Sigma m}}, \\
\varphi_{x}=2 \operatorname{arcctg} \frac{U_{\Sigma m}}{U_{R m}}, \\
\varphi_{x}=2 \arcsin \frac{U_{R m}}{2 U_{m}}, \\
\varphi_{x}=2 \arccos \frac{U_{\Sigma m}}{2 U_{m}} .
\end{gathered}
$$

\section{TASK}

Inequality of amplitudes leads to a measurement error. Since this effect is not obvious, the purpose of this article is to assess the impact of amplitudes inequality on the phase shift measurement accuracy with the help of above mentioned algorithmic methods. In addition, many tasks in science and technology are solved by measuring small phase shifts. Thus, it is important to define the most appropriate methods for measuring small phase shifts.

\section{MATERIAL PRESENTING}

Here we are considering the situation with unequal amplitudes of signals, so let's write the expression for two harmonic signals with different amplitudes

$$
u_{1}^{\prime}(t)=U_{1} \sin \omega t=U_{m} \sin \omega t
$$

$$
u_{2}^{\prime}(t)=U_{2} \sin \left(\omega t+\varphi_{x}\right)=U_{m}(1+\delta U) \sin \left(\omega t+\varphi_{x}\right) .
$$

In this case, by adding the signals for summary signal we get

$$
\begin{aligned}
& u_{\Sigma}^{\prime}(t)=u_{1}^{\prime}(t)+u_{2}^{\prime}(t)=U_{m} \sqrt{4 \cos ^{2} \frac{\varphi_{x}}{2}(1+\delta U)+(\delta U)^{2}} \times \\
& \times \sin \left\{\omega t+\frac{\varphi_{x}}{2}+\operatorname{arctg}\left[\frac{\delta U}{(2+\delta U)} \cdot \operatorname{tg} \frac{\varphi_{x}}{2}\right]\right\}
\end{aligned}
$$

In a similar way for difference signal we get

$$
\begin{aligned}
& u_{R}^{\prime}(t)=u_{2}^{\prime}(t)-u_{1}^{\prime}(t)=U_{m} \sqrt{4 \sin ^{2} \frac{\varphi_{x}}{2}(1+\delta U)+(\delta U)^{2}} \times \\
& \times \sin \left\{\omega t+\frac{\pi}{2}+\frac{\varphi_{x}}{2}-\operatorname{arctg}\left[\frac{\delta U}{(2+\delta U)} \cdot \operatorname{tg}\left(\frac{\pi}{2}-\frac{\varphi_{x}}{2}\right)\right]\right\}
\end{aligned}
$$

Now let us define the moment $t_{1}^{\prime}$ of summary signal transition (5) through zero, because at this moment measuring of instantaneous value of the difference signal will be accomplished. Under this condition we get

$$
t_{1}^{\prime}=\left\{-\frac{\varphi_{x}}{2}-\operatorname{arctg}\left[\frac{\delta U}{(2+\delta U)} \cdot \operatorname{tg} \frac{\varphi_{x}}{2}\right]\right\} / \omega .
$$

Let us define the value of the different voltage at the moment $t_{1}^{\prime}$

$$
\begin{aligned}
& u_{R}^{\prime}\left(t_{1}^{\prime}\right)=U_{m} \sqrt{4 \sin ^{2} \frac{\varphi_{x}}{2}(1+\delta U)+(\delta U)^{2}} \times \sin \left\{\frac{\pi}{2}-\right. \\
& \left.-\operatorname{arctg}\left[\frac{\delta U}{(2+\delta U)} \cdot \operatorname{tg} \frac{\varphi_{x}}{2}\right]-\operatorname{arctg}\left[\frac{\delta U}{(2+\delta U)} \cdot \operatorname{tg}\left(\frac{\pi}{2}-\frac{\varphi_{x}}{2}\right)\right]\right\}
\end{aligned}
$$

We shall define from (6) the moment $t_{2}^{\prime}$ of difference signal transition through zero because at this moment measuring of instantaneous value of summary signal will be accomplished. To get intervals of summary and difference signals with the same operators we should take into account that the second transition of function $\sin$ through zero is at the distance $\pi$ from the first one, so we get

$$
t_{2}^{\prime}=\left\{\frac{\pi}{2}-\frac{\varphi_{x}}{2}+\operatorname{arctg}\left[\frac{\delta U}{(2+\delta U)} \cdot \operatorname{tg}\left(\frac{\pi}{2}-\frac{\varphi_{x}}{2}\right)\right]\right\} / \omega .
$$

Summary signal value at the moment $t_{2}^{\prime}$ will be

$$
\begin{gathered}
u_{\Sigma}^{\prime}\left(t_{2}^{\prime}\right)=U_{m} \sqrt{4 \cos ^{2} \frac{\varphi_{x}}{2}(1+\delta U)+(\delta U)^{2}} \times \\
\times \sin \left\{\frac{\pi}{2}+\operatorname{arctg}\left[\frac{\delta U}{(2+\delta U)} \cdot \operatorname{tg} \frac{\varphi_{x}}{2}+\right.\right. \\
\left.+\operatorname{arctg}\left[\frac{\delta U}{(2+\delta U)} \cdot \operatorname{tg}\left(\frac{\pi}{2}-\frac{\varphi_{x}}{2}\right]\right]\right\} .
\end{gathered}
$$

\section{A. First Method Errors}

Let us write down the expression for the phase shift similarly to the expression (1) 


$$
\begin{gathered}
\varphi_{x}^{\prime}=2 \operatorname{arctg} \frac{u_{R}^{\prime}\left(t_{1}^{\prime}\right)}{u_{\Sigma}^{\prime}\left(t_{2}^{\prime}\right)}=2 \operatorname{arctg}\left\{\sqrt{\frac{4 \sin ^{2} \frac{\varphi_{x}}{2}(1+\delta U)+(\delta U)^{2}}{4 \cos ^{2} \frac{\varphi_{x}}{2}(1+\delta U)+(\delta U)^{2}}} \times\right. \\
\left.\times \frac{\sin \left\{\frac{\pi}{2}-\operatorname{arctg}\left[\frac{\delta U}{(2+\delta U)} \cdot \operatorname{tg} \frac{\varphi_{x}}{2}-\operatorname{arctg}\left[\frac{\delta U}{(2+\delta U)} \cdot \operatorname{tg}\left(\frac{\pi}{2}-\frac{\varphi_{x}}{2}\right)\right]\right\}\right.}{\sin \left\{\frac{\pi}{2}+\operatorname{arctg}\left[\frac{\delta U}{(2+\delta U)} \cdot \operatorname{tg} \frac{\varphi_{x}}{2}+\operatorname{arctg}\left[\frac{\delta U}{(2+\delta U)} \cdot \operatorname{tg}\left(\frac{\pi}{2}-\frac{\varphi_{x}}{2}\right]\right]\right\}\right.}\right\} \\
=2 \operatorname{arctg} \sqrt{\frac{4 \sin ^{2} \frac{\varphi_{x}}{2}(1+\delta U)+(\delta U)^{2}}{4 \cos ^{2} \frac{\varphi_{x}}{2}(1+\delta U)+(\delta U)^{2}}} .
\end{gathered}
$$

Herewith absolute measurement error will be

$$
\Delta \varphi=\varphi_{x}^{\prime}-\varphi_{x}=2 \operatorname{arctg} \sqrt{\frac{4 \sin ^{2} \frac{\varphi_{x}}{2}(1+\delta U)+(\delta U)^{2}}{4 \cos ^{2} \frac{\varphi_{x}}{2}(1+\delta U)+(\delta U)^{2}}}-\varphi_{x} .
$$

Let us define from (7) the error relative value

$$
\delta \varphi=\frac{\Delta \varphi}{\varphi_{x}} \cdot 100 \%=\left\{\frac{2 \operatorname{arctg} \sqrt{\frac{4 \sin ^{2} \frac{\varphi_{x}}{2}(1+\delta U)+(\delta U)^{2}}{4 \cos ^{2} \frac{\varphi_{x}}{2}(1+\delta U)+(\delta U)^{2}}}}{\varphi_{x}}-\right.
$$

Dependence of the relative error (8) on the phase shift for different values $\delta U$ is shown in Fig. 1 .

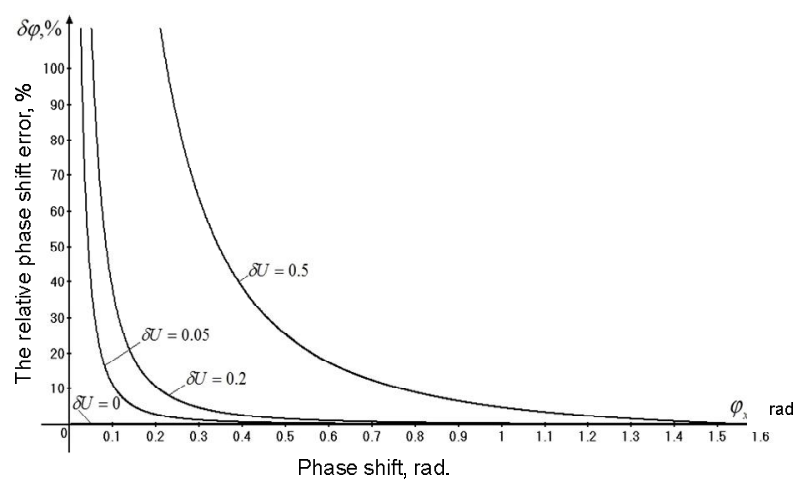

Fig. 1. Dependence of the relative error on the phase shift for different values $\delta U$

In (8) and Fig. 1 we can see that the phase measurement error according to this method does not depend on extra phase shifts of difference and summery signals, caused by the signals amplitudes inequality but depends on amplitudes inequality only. When $\delta U=100 \%$ we can define the phase shift which corresponds to sensitivity threshold (from 0.03 to 0.3 ) red for considered values $\delta U$.

Thus, it is clear that the increasing in $\delta U$ results in the sensitivity threshold increase too. Therefore, this method is unsuited for measuring small phase shifts.

\section{B. Second Method Errors}

For the second method, described by

$$
\varphi_{x}=2 \operatorname{arcctg} \frac{U_{\sum m}}{U_{R m}},
$$

we receive the following correlation:

$$
\varphi_{x}^{\prime}=2 \operatorname{arcctg} \frac{u_{\Sigma}^{\prime}\left(t_{2}^{\prime}\right)}{u_{R}^{\prime}\left(t_{1}^{\prime}\right)}=2 \operatorname{arcctg} \sqrt{\frac{4 \cos ^{2} \frac{\varphi_{x}}{2}(1+\delta U)+(\delta U)^{2}}{4 \sin ^{2} \frac{\varphi_{x}}{2}(1+\delta U)+(\delta U)^{2}}} .
$$

Absolute measurement error will be

$$
\Delta \varphi=\varphi_{x}^{\prime}-\varphi_{x}=2 \operatorname{arcctg} \sqrt{\frac{4 \cos ^{2} \frac{\varphi_{x}}{2}(1+\delta U)+(\delta U)^{2}}{4 \sin ^{2} \frac{\varphi_{x}}{2}(1+\delta U)+(\delta U)^{2}}}-\varphi_{x} \cdot
$$

From the expression (9) we are going to define the relative value of error

$$
\delta \varphi=\frac{\Delta \varphi}{\varphi_{x}} \cdot 100 \%=\left(\frac{2 \operatorname{arcctg} \sqrt{\frac{4 \cos ^{2} \frac{\varphi_{x}}{2}(1+\delta U)+(\delta U)^{2}}{4 \sin ^{2} \frac{\varphi_{x}}{2}(1+\delta U)+(\delta U)^{2}}}}{-1) \cdot 100 \% .}-\right.
$$

Fig. 2 represents the graph of dependence (10) on the phase shift $\varphi_{x}$ for different values $\delta U$.

From the Fig. 2 it is clear that the sensitivity threshold for this method ranges from $0,03 \mathrm{rad}$. (when $\delta U=0,05$ ) to $0,23 \mathrm{rad}$. (when $\delta U=0,5$ ). It's possible to make a conclusion that this method is also unsuited for small phase shifts measuring because additive component of error takes place and so the sensitivity threshold is too high.

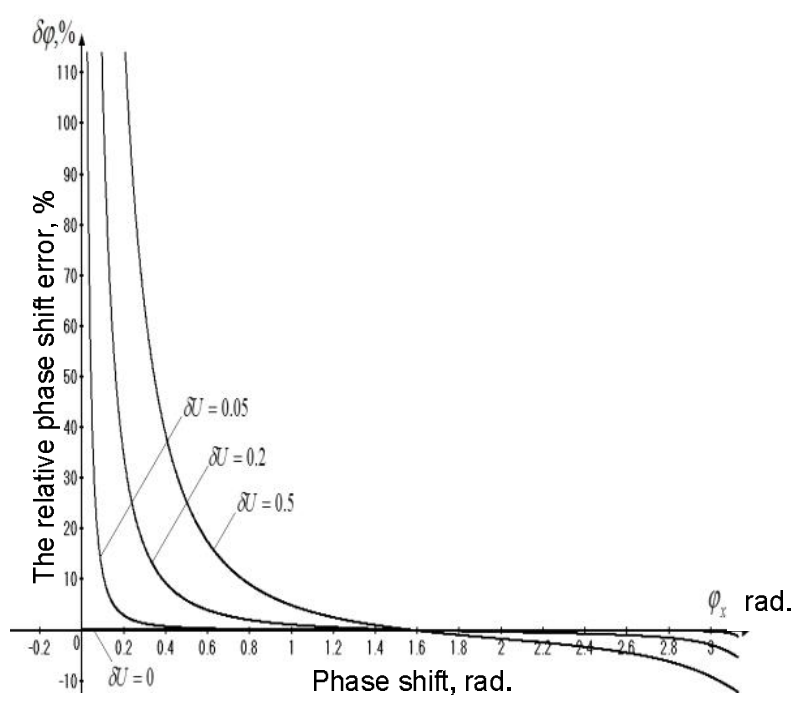

Fig. 2. Dependence of error $\delta \varphi$ on phase shift for different values $\delta U$

\section{Third Method Errors}

If the phase shift is defined with the expression (3)

$$
\varphi_{x}=2 \arcsin \frac{U_{R m}}{2 U_{m}},
$$

we will get the following expression for the signals with unequal amplitudes 


$$
\begin{aligned}
\varphi_{x}^{\prime}=2 \arcsin & \frac{u_{R}^{\prime}\left(t_{1}^{\prime}\right)}{2 U_{m}}=2 \arcsin \left(\sqrt{4 \sin ^{2} \frac{\varphi_{x}}{2}(1+\delta U)+(\delta U)^{2}} \times\right. \\
\times & \sin \left\{\frac{\pi}{2}-\operatorname{arctg}\left[\frac{\delta U}{(2+\delta U)} \cdot \operatorname{tg} \frac{\varphi_{x}}{2}\right]-\right. \\
& \left.\left.-\operatorname{arctg}\left[\frac{\delta U}{(2+\delta U)} \cdot \operatorname{tg}\left(\frac{\pi}{2}-\frac{\varphi_{x}}{2}\right)\right]\right\} / 2\right) .
\end{aligned}
$$

Absolute measurement error will be

$$
\begin{aligned}
& \Delta \varphi=\varphi_{x}^{\prime}-\varphi_{x}=2 \arcsin \left(\frac{1}{2} \sqrt{4 \sin ^{2} \frac{\varphi_{x}}{2}(1+\delta U)+(\delta U)^{2} \times}\right. \\
& \times \sin \left\{\frac{\pi}{2}-\operatorname{arctg}\left[\frac{\delta U}{(2+\delta U)} \cdot \operatorname{tg} \frac{\varphi_{x}}{2}\right]-\right. \\
&\left.\left.-\operatorname{arctg}\left[\frac{\delta U}{(2+\delta U)} \cdot \operatorname{tg}\left(\frac{\pi}{2}-\frac{\varphi_{x}}{2}\right)\right]\right\}\right)-\varphi_{x} .
\end{aligned}
$$

From the expression (11) let us define the relative value of the error

$$
\begin{gathered}
\delta \varphi=\frac{\Delta \varphi}{\varphi_{x}} \cdot 100 \%=2 \arcsin \frac{u_{R}^{\prime}\left(t_{1}^{\prime}\right)}{2 U_{m}} \cdot 100 \%= \\
=\frac{2}{\varphi_{x}} \arcsin \left(\frac{1}{2} \sqrt{4 \sin ^{2} \frac{\varphi_{x}}{2}(1+\delta U)+(\delta U)^{2}} \times\right. \\
\times \sin \left\{\frac{\pi}{2}-\operatorname{arctg}\left[\frac{\delta U}{(2+\delta U)} \cdot \operatorname{tg} \frac{\varphi_{x}}{2}\right]-\right. \\
\left.\left.\left.-\operatorname{arctg}\left[\frac{\delta U}{(2+\delta U)} \cdot \operatorname{tg}\left(\frac{\pi}{2}-\frac{\varphi_{x}}{2}\right)\right]\right\}\right)-1\right) \cdot 100 \% .
\end{gathered}
$$

The graph of dependence (12) on phase shift $\varphi_{x}$ for different values $\delta U$ is represented in Fig. 3.

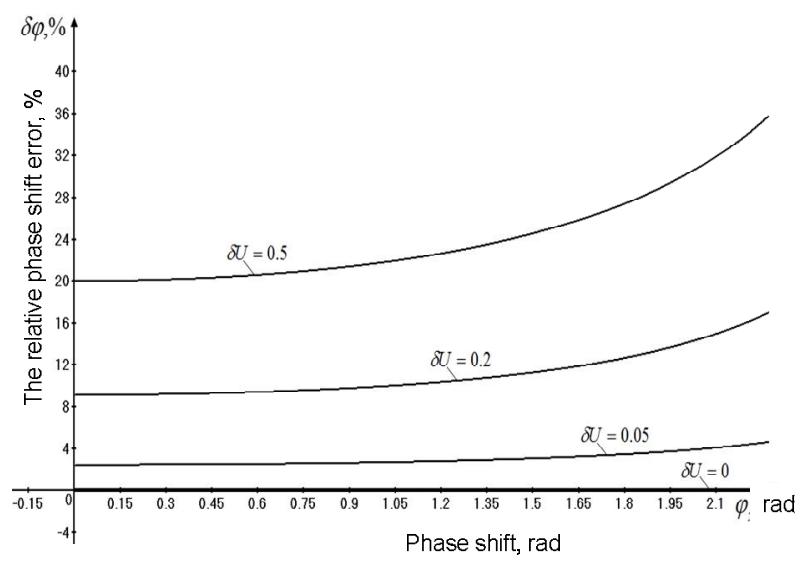

Fig. 3. Dependence of relative error on phase shift for different values $\delta U$

Fig. 3 shows that multiplicative component of the error and nonlinear complex take place. Thus, this method can provide the smallest sensitivity threshold.

\section{Fourth Method Errors}

In case the phase shift is defined similarly to the expression (4)

$$
\varphi_{x}=2 \arccos \frac{U_{\sum m}}{2 U_{m}},
$$

the expression for phase shift becomes

$$
\begin{aligned}
\varphi_{x}^{\prime}=2 \arccos & \frac{u_{\Sigma}^{\prime}\left(t_{2}^{\prime}\right)}{2 U_{m}}=2 \arccos \left(\frac{1}{2} \sqrt{4 \cos ^{2} \frac{\varphi_{x}}{2}(1+\delta U)+(\delta U)^{2}} \times\right. \\
& \times \sin \left\{\frac{\pi}{2}+\operatorname{arctg}\left[\frac{\delta U}{(2+\delta U)} \cdot \operatorname{tg} \frac{\varphi_{x}}{2}\right]+\right. \\
& \left.\left.+\operatorname{arctg}\left[\frac{\delta U}{(2+\delta U)} \cdot \operatorname{tg}\left(\frac{\pi}{2}-\frac{\varphi_{x}}{2}\right)\right]\right\}\right) .
\end{aligned}
$$

Absolute measurement value will be

$$
\begin{gathered}
\Delta \varphi=\varphi_{x}^{\prime}-\varphi_{x}=2 \arccos \left(\frac{1}{2} \sqrt{4 \cos ^{2} \frac{\varphi_{x}}{2}(1+\delta U)+(\delta U)^{2}} \times\right. \\
\times \sin \left\{\frac{\pi}{2}+\operatorname{arctg}\left[\frac{\delta U}{(2+\delta U)} \cdot \operatorname{tg} \frac{\varphi_{x}}{2}\right]+\right. \\
\left.\left.+\operatorname{arctg}\left[\frac{\delta U}{(2+\delta U)} \cdot \operatorname{tg}\left(\frac{\pi}{2}-\frac{\varphi_{x}}{2}\right)\right]\right\}\right)-\varphi_{x} .
\end{gathered}
$$

From the expression (13) let us define the relative value of error

$$
\begin{gathered}
\delta \varphi=\frac{\Delta \varphi}{\varphi_{x}} \cdot 100 \%=\left(\frac { 2 } { \varphi _ { x } } \operatorname { a r c c o s } \left(\frac{1}{2} \sqrt{4 \cos ^{2} \frac{\varphi_{x}}{2}(1+\delta U)+(\delta U)^{2}} \times\right.\right. \\
\times \sin \left\{\frac{\pi}{2}+\operatorname{arctg}\left[\frac{\delta U}{(2+\delta U)} \cdot \operatorname{tg} \frac{\varphi_{x}}{2}\right]+\right. \\
\left.\left.\left.+\operatorname{arctg}\left[\frac{\delta U}{(2+\delta U)} \cdot \operatorname{tg}\left(\frac{\pi}{2}-\frac{\varphi_{x}}{2}\right)\right]\right\}\right)-1\right) \cdot 100 \% .
\end{gathered}
$$

The graph of dependence (14) for different values is represented in Fig. 4.

From Fig. 4. we can see that this method is characterized by a significant sensitivity threshold (from $0.3 \mathrm{rad}$. when $\delta \mathrm{U}=0,05$ to $0.85 \mathrm{rad}$. when $\delta=0,5$ ) because the additive component of error takes place and can change its operator and has a point of discontinuity of the first kind. These break points occur at the level of accuracy which equals sensitivity threshold and at the value of measured phase shift, which increases when relative inequality of amplitude signals increases. Therefore, this method is unsuitable for measuring not only small phase shifts, but large ones too.

Fig. 5 represents a dependence of method error (3) on the relative inequality of amplitudes of compared signals.

From the Fig. 5 we can see that the additive component of method is absent so the inequality of amplitudes doesn't influence the sensitivity threshold. Therefore, this method does not require to use special measures for alignment signal amplitudes. It can be considered the best for measuring small and large phase shifts.

\section{E. Existing Amplitude Independent Method}

An independent amplitude phase shift measurement method is known [6]. It measures amplitude values of one and the other signal. The amplitude value of the first signal is reduced by $K$ times. The resulting voltage is compared with the instantaneous value of the same signal. 


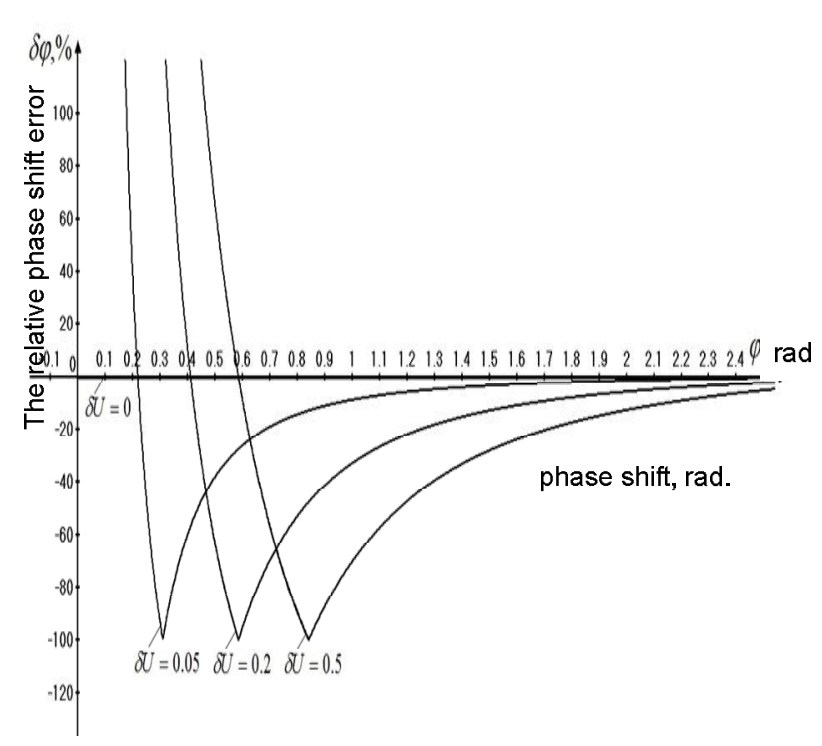

Fig. 4. Graph of (14) for different values $\delta U$

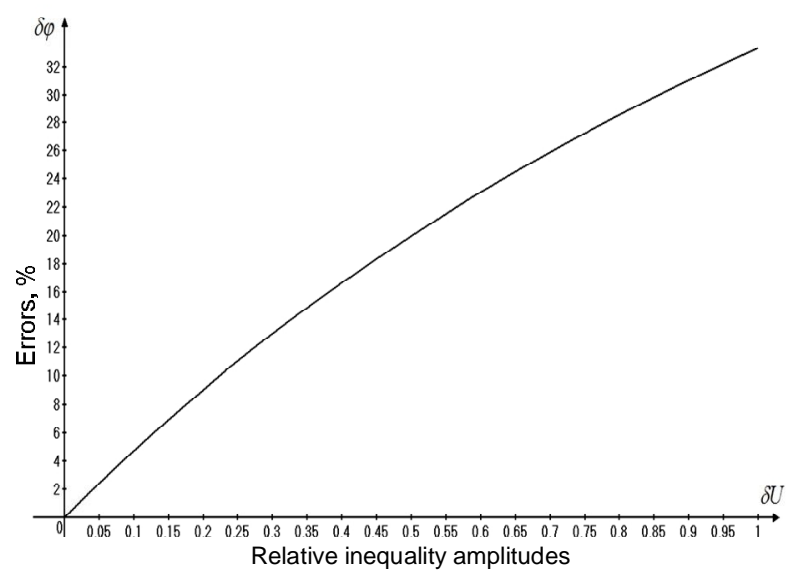

Fig. 5. Dependence of method error (3) from the relative inequality of amplitudes of compared signals

At the moment when these values are equal, that is

$$
t_{1}=\frac{1}{\omega} \arcsin \frac{1}{K}
$$

measure the value of the second signal $u_{2}\left(t_{1}\right)$. The value of the phase shift is found by the formula [5]

$$
\varphi_{x}=\arcsin \frac{1}{K}-\arcsin \left(\frac{u_{2}\left(t_{1}\right)}{U_{m 2}}\right) .
$$

It uses an indirect method of measuring the phase shift. Phase shift measurement error is determined by errors of measuring of three voltages and one comparison. This method is also difficult to implement.

\section{F. Proposed amplitude independent methods}

We proposed a simpler and more accurate amplitude independent way to measure phase shift between the harmonic signals. It does not require signal amplitudes alignment.
Here, for measuring the phase shift between two harmonic signals

$$
u_{1}(t)=U_{m 1} \sin \left(\omega t+\varphi_{x}\right)
$$

and

$$
u_{2}(t)=U_{m 2} \sin \omega t
$$

We should measure the amplitude $U_{m 1}$ of the first signal and the instantaneous value $u_{1}\left(t_{0}\right)$ of the same signal. The moment $t_{0}$ is a moment when the instantaneous value $u_{2}\left(t_{0}\right)$ of the second signal is zero. Then the phase shift $\varphi_{x}$ is found by the formula

$$
\varphi_{x}=\arcsin \frac{u_{1}\left(t_{0}\right)}{U_{m 1}} .
$$

It is, therefore, necessary to make only two measurements of instantaneous voltage values. This allows you to determine the phase shift with higher accuracy and simplifies the implementation process.

Fig. 6 shows a block scheme of a device that works in this way.

The device consists of a differentiator DF, comparators $\mathrm{C} 1$ and $\mathrm{C} 2$, and monostable multivibrators MM1 and MM2, logic circuit LC OR, microcontroller MC, which includes analog-to-digital converter ADC and memory RAM, and a display.

Time diagram of the device is shown in Fig. 7.

The device operates as follows. The input of differentiator DF1 and measuring input of the ADC of microcontroller $\mathrm{MC}$ enters signal $u_{i n 1}(t)$. The input signal $u_{\text {in } 2}(t)$ is fed to the input of the comparator $\mathrm{K} 2$. The signal $u_{i n 1}(t)$ by differentiator DF1 shifts the phase to $\frac{\pi}{2}$ and then comparator $\mathrm{K} 1$ converts it into a rectangular signal $u_{2}(t)$ (Fig. 7). The fronts $u_{2}(t)$ coincide with the moment at which $u_{i n 1}(t)$ has the signal amplitude value $U_{m 1}$. Monostable multivibrator MM1 from rectangular signal $u_{2}(t)$ generates short pulses (Fig. 7). They come to the first input logic circuit OR and through its output $u_{4}(t)$ (Fig. 6) to run to launch input of the ADC microcontroller's MC. At this moment in measuring input of the ADC signal $u_{i n 1}(t)$ has a peak value $U_{m 1}$, which is written after the $\mathrm{ADC}$ in the microcontroller's RAM memory cell MC (Fig. 6).

At the same time the input of the comparator $\mathrm{K} 2$ is fed with the second signal $u_{i n 2}(t)$. Comparator K2 converts it into a rectangular signal $u_{5}(t)$ (Fig. 7), which fronts coincide with moments $t_{0}$ equal to zero signal $u_{6 x 2}\left(t_{0}\right)=0$. Monostable multivibrator MM2 with rectangular signal $u_{5}(t)$ generates short pulses $u_{6}(t)$ (Fig. 7). These pulses are fed through the second logic circuit OR entrance and its exit to launch ADC. In measuring ADC entrance at this moment an instant 
value $u_{1}\left(t_{0}\right)$ is acting, which is recorded into the second memory cell microcontroller's MC. On the basis of the data recorded in the cells, microcontroller MC calculates the phase shift according to the formula (1), which is shown on the display.

Thus, errors of compared signal amplitude inequality when measuring the phase shift between them are eliminated. This makes it possible to increase the accuracy by reducing the number of auxiliary measurements and simplify the implementation of phase shift measurement.

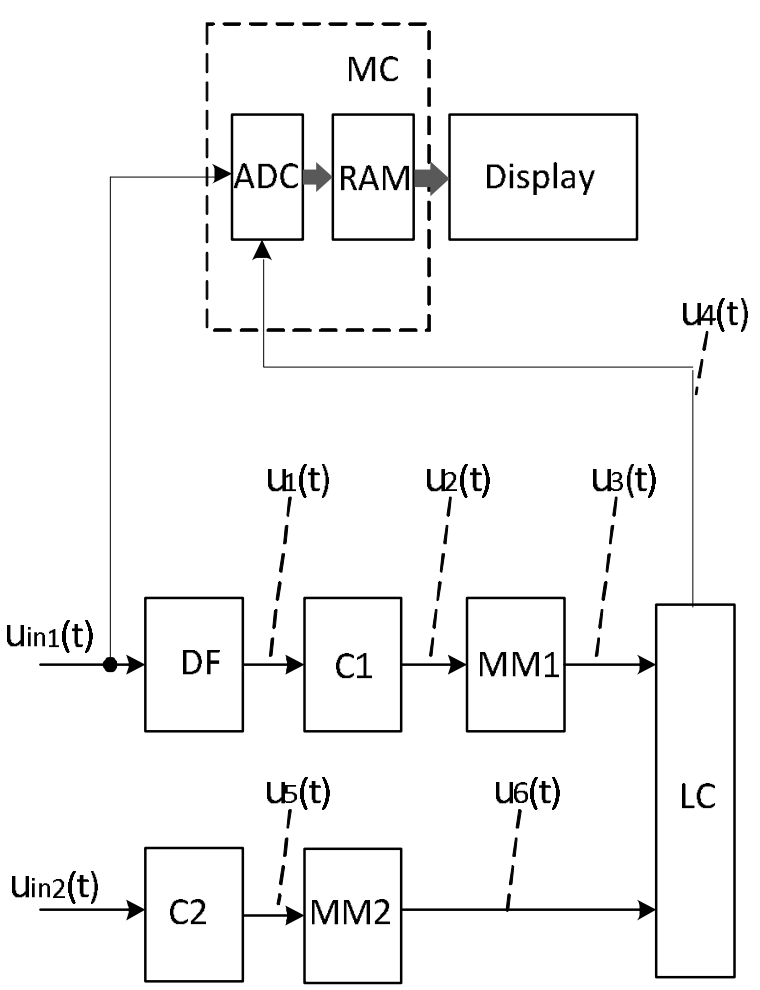

Fig. 6. Block scheme of measurer the phase shift

\section{G. The method of increasing sensitivity}

Sometimes it may be necessary to have ten times higher sensitivity. When the amplitude of the second signal is close to the upper level of dynamic range and the amplitude of the first one is several times smaller, the attempt to obtain the desired increase of sensitivity with the help of the method will fail. Here you can increase the sensitivity by only a few times, that is how the first signal can be enhanced.

At low frequencies to increase the sensitivity by $K$ times, you can proceed according to the following algorithm:

1) align signal amplitude without changing their initial phases;

2) receive the difference signal $u_{1}(t)-u_{2}(t)$;

$3)$ enhance the difference signals by $(K+\Delta K)$ times

$$
(K+\Delta K)\left[u_{1}(t)-u_{2}(t)\right]
$$

4) do sampling of difference signals

$$
(K+\Delta K)\left[u_{1}\left(t_{0}\right)-u_{2}\left(t_{0}\right)\right]=(K+\Delta K) \cdot u_{1}\left(t_{0}\right)
$$

at that time $t_{0}$, when the instantaneous value of the second signal $u_{2}\left(t_{0}\right)$ is zero;

5 ) do sampling of peak value $U_{m 1}$ of signal $u_{1}(t)$;

6) calculate the phase shift according to the formula

$$
\varphi_{x}=\arcsin \frac{(K+\Delta K) \cdot u_{1}\left(t_{0}\right)}{k U_{m 1}}
$$

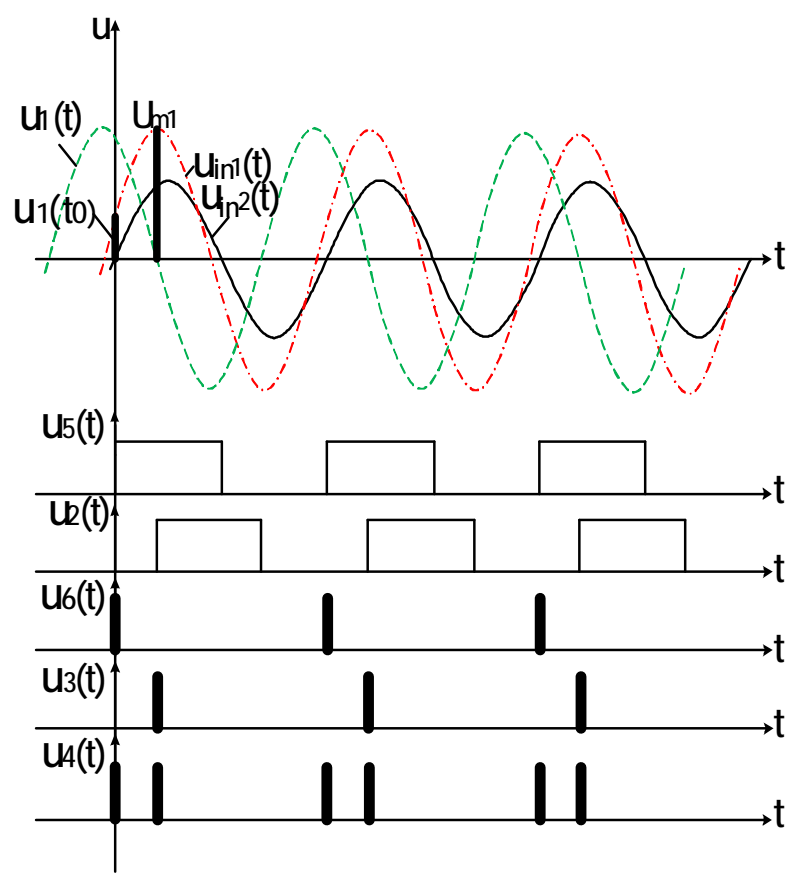

Fig. 7. Time diagram of the device

To implement this algorithm is possible by means of the block diagram Fig. 8.

The meter according to Fig. 8 consists of amplitude equalization scheme AES, difference scheme DS, amplifier A, differentiator DF, comparators $\mathrm{C} 1$ and $\mathrm{C} 2$, monostable multivibrator MM1 and MM2, logic circuit LC OR, microcontroller MC, with embedded analog-todigital converter ADC and memory RAM, and display.

Schemes Fig. 8 and Fig. 6 differ from one another by introduction of several additional blocks: amplitude equalization scheme AES, difference amplifier circuit DAC and amplifier A.

The need for these schemes has been justified above. In particular, the AES aligns the signal amplitudes without changing their initial phases. Difference scheme DS forms the difference of output AES signals. This difference is amplified by the amplifier $\mathrm{A}$ and fed to the second digital input of the microcontroller MC. This scheme provides a measurement of the instantaneous value of the first signal from the output CBA at the moments when the second signal is zero, and when the first signal is amplitude value. Then the formula (16) is 
used to calculate the phase shift. This approach allows to increase sensitivity which is needed when measuring small phase shifts. In such a structure there are used standard units whose work is understandable. The only unit whose work may raise questions, is amplitude equalization scheme AES.

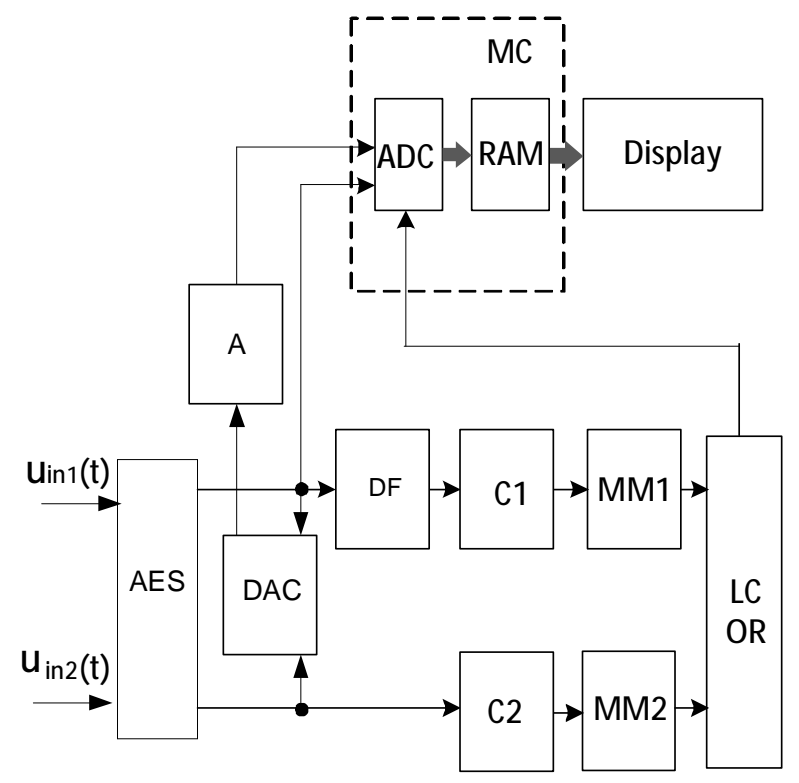

Fig. 8. Block diagram of measuring the phase shift of hypersensitivity

Therefore, we are going to consider a variant of the block diagram of the AES (Fig. 9).

The scheme consists of two managed voltage dividers VDM1 and VDM2, two rectifiers R1 and R2, two low-pass filters LPF1 and LPF2, difference scheme DS adder AD. VDM in its simplest form can consist of active resistor and resistor voltage-controlled, which may be, for example, transistor FET.

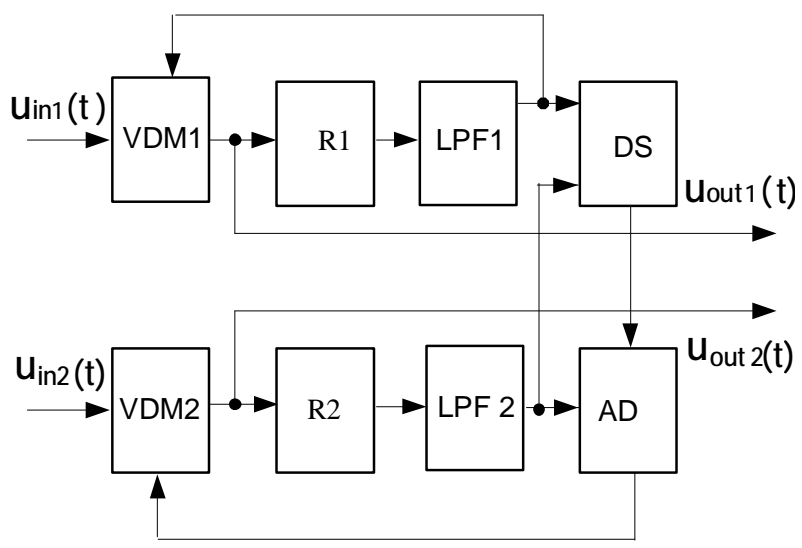

Fig. 9. The structure of the signal amplitudes equalization scheme

The scheme Fig. 9 shows that the output signals VDM1 VDM2 are straightened The filters LPF1 and
LPF2 extract permanent components from them. One of them from output of LPF1 is fed to the control input VDM1 directly. But at the same time it is sent to one of the difference scheme DS entrance. The second component from LPF2exit is fed to the second circuit DS entrance and at the same time - to the second the adder $\mathrm{AD}$ entrance. At the first adder $\mathrm{AD}$ entrance voltage is supplied from the difference circuit DS exit. The adder AD exit constant voltage is sent to controlling VDM2 entrance.

If the amplitude of the output signals VDM1 and VDM2 are not equal, the voltage proportional to this difference from the exit of the adder circuit AD is added to the exit voltage filter LPF2. By reaching VDM2 controlling entrance it changes its transmission ratio so that it helps equalize output signals amplitudes of VDM1 and VDM2.

\section{CONCLUSIONS}

Taking into consideration the obtained expressions and graphs for the relative error caused by signals amplitudes inequality, we may conclude that for small phase shifts measurement the best method is the one described in (3). From Fig. 3 and Fig. 5 we can see that the error of the method has nonlinear and multiplicative components which do not influence the sensitivity threshold. At the same time, for all other methods amplitudes inequality leads to a significant additive component of the error which increases with amplitude difference of compared signals increase and limits the sensitivity threshold.

It has been shown that the error of compared signal amplitude inequality when measuring the phase shift between them can be eliminated. The scheme of the device is easy to implement. An improved accuracy is achieved by reducing the number of auxiliary measurements.

Thus, to achieve a high sensitivity and accuracy of measuring small phase shifts by algorithmic sumdifference method there are several possibilities: 1) use third method for measuring the phase shift by the formula (3). I that case errors from inequality of amplitudes have multiplicative effect on the sensitivity threshold; 2) use simple amplitudes independent method, implemented according to the scheme Fig. 6; 3) if necessary to have a higher sensitivity the method implemented in the scheme Fig. 8. can be applied.

\section{REFERENCES}

[1] Melnyk A. O. Cyber-Physical Systems: Problems of Creation and Directions of Development. Visnyk National University "Lviv Polytechnic" Computer systems and networks, number 806, 2014. - P. 154-161.

[2] David G., Michael B. David G., "Introduction to mechatronics and measurement systems", McGraw-Hill, 2007.

[3] I. Buchma, "Development of non-destructive testing of steel sheet structures using eddy current diagnostics" "DRE-2008". International Scientific Conference, Technical University, Polish, Rep. pp. 59-62, Opole May 19-21, 2008. 
[4] I. Buchma, L. Mychajlovych"Reducing the sensitivity threshold of low frequency single-channel path thickness gauge steel sheet structures" Methods and instruments for quality control, No. 10, P. 22-23, 2003.

[5] I. Buchma , Y. Vynnychek, "Comparison of algorithmic sumodifference methods for measuring the phase shift", ACSN-13, International Conference, Technical University "Lvivska Politehnika“, Lviv, Ukraine, pp. 187-188, 2013.

[6] Kondratov V. T., "Algorithmic methods for measuring the instantaneous values of infra-low signals phase shift" Glushkov's Institute of kibernetic., Kyiv, Preprint 04-47, pp. 40, 1984

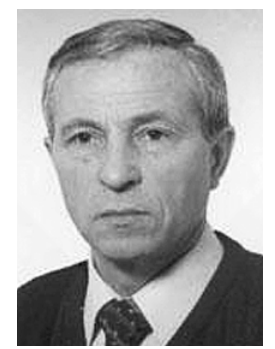

I. Buchma was born in Lviv, Ukraine, in 1942. He obtained engineer degree in electronics engineering at Lviv Polytechnic Institute, Lviv, Ukraine, in 1964. From 1965 to 1970 he was an engineer at an electronic devices factory in Lviv. From 1970 to 1980 he did a postgraduate degree and was a research assistant at Physic-Mechanical Institute of the Academy of Sciences of Ukraine in Lviv. He was awarded his Ph.D. in measuring technology of electrical and magnetic quantities at Physic-Mechanical Institute in 1978.

From 1980 to 2002 he was an assistant professor with the National University "Lviv Polytechnic" in Lviv. In 2001 he defended his doctoral thesis. In 2004 he was awarded the title of professor. From 2002 to 2013 he was a Professor of National University "Lviv Polytechnic" in Lviv, Ukraine, and Technical University in Opole, Poland. Since 2013 he has been a Professor of National University "Lviv Polytechnic". He is the author of three books, more than 100 scientific articles, and more than 50 inventions. His scientific interests include research of inhomogeneous environments by electromagnetic methods.

\section{ACKNOWLEDGEMENT}

The scientific results, presented in this article, were obtained within the frame of research project number 0115U000446, 01.01.2015-31.12.2017, financially supported by the Ministry of Education and Science of Ukraine. 